\title{
18. Fortbildungskongress
}

\section{Fortschritte der Allergologie, Immunologie und Dermatologie}

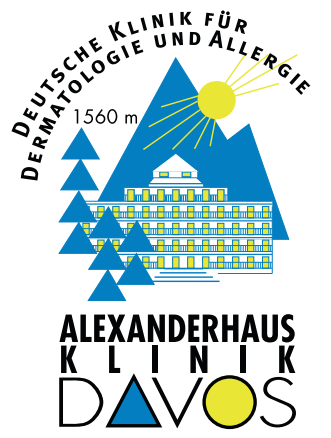

mit

\section{Dreiländertreffen „Allergologie“}

\section{der Deutschen, Schweizerischen und Österreichischen Gesellschaften für Allergologie und klinische Immunologie}

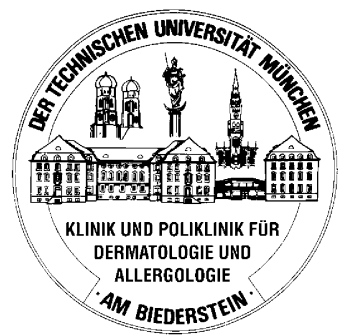

Kongress-Zentrum Davos, 4.-7. September 2002

Veranstalter: Klinik und Poliklinik für Dermatologie und Allergologie am Biederstein, TeChNische Universität München, Und Deutsche Klinik für Dermatologie Und Allergie Davos, Alexanderhausklinik, Davos Platz

Wissenschaftliche Leitung: Prof. Dr. Dr. Johannes Ring, München, und Prof. Dr. Dr. SiEgFried Borelli, Davos

\section{Grußwort}

Sehr geehrte Frau Kollegin!

Sehr geehrter Herr Kollege!

Allergien und umweltbedingte Erkrankungen treten immer mehr in den Vordergrund. Neue Erkenntnisse in Diagnostik und Therapie sind deshalb eine wichtige Voraussetzung, um den steigenden Bedürfnissen bei der Behandlung unserer Patienten gerecht zu werden.

In diesem Jahr werden wir den 18. Fortbildungskongress „Fortschritte der Allergologie, Immunologie und Dermatologie" wieder zusammen mit dem Dreiländertreffen der Deutschen, Österreichischen und Schweizerischen Gesellschaften für Allergologie und klinische Immunologie veranstalten. Wir freuen uns auf den Austausch mit unseren benachbarten Kollegen!

Aktuelle Themen wie Allergie-Diagnostik und -Therapie, Allergie und Umwelt, Allergien im

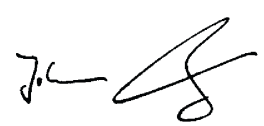

Prof. Dr. med. Dr.phil. J. Ring
Kindesalter, Nahrungs- und Arzneimittelallergien, Neurodermitis / atopisches Ekzem, dermatologische Kosmetologie, Hochgebirgsklimatherapie und Rehabilitationsmedizin werden in Vorträgen, Symposien und Seminaren abgehandelt werden. Im Rahmen der täglich stattfindenden Diaklinik werden ausgewählte Kasuistiken vorgestellt und diskutiert. Um unseren jungen Wissenschaftlern die aktive Teilnahme zu ermöglichen, werden wir in diesem Jahr auch eine Posterausstellung organisieren.

Die Klinik und Poliklinik für Dermatologie und Allergologie am Biederstein der Technischen Universität München veranstaltet diesen Kongress gemeinsam mit der traditionsreichen Deutschen Klinik für Dermatologie und Allergie Davos - Alexanderhausklinik. Wir freuen uns, Sie im Davoser Hochgebirgstal begrüssen zu dürfen!

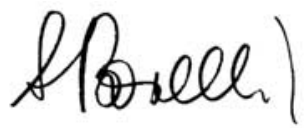

Prof. Dr. med. Dr.phil. S. Borelli 